\title{
Identification of a spatial linear model based on earthquake-induced data and genetic algorithm with parallel selection
}

\author{
David Hicham Bassir ${ }^{1, a}$, José Luis Zapico ${ }^{2}$, María Placeres González ${ }^{2}$ and Rodolfo Alonso ${ }^{2}$ \\ 1 Institute of FEMTO-ST, UMR / CNRS 6174, Dept. LMARC, 24, rue de l'Epitaphe, 25000 Besançon, France \\ 2 Universidad de Oviedo, Departamento de Construcción e Ingeniería de Fabricación, Campus de Gijón, 33203 Gijón, Spain
}

Received 6 May 2007; accepted 9 July 2007

\begin{abstract}
This paper deals with the parametric identification of a small-scale bridge model that was intended to approximately reproduce the transversal dynamic behaviour of its corresponding prototype. This is representative of a typical multi-span continuous-deck irregular bridge. A linear model with viscous damping is proposed to reproduce its dynamic response. The identification is carried out in the time domain using experimental earthquake-induced data and assuming the mass is known. An identification procedure including a genetic algorithm with parallel selection has been developed. In the studied case, the procedure has demonstrated to be robust. It is shown that the model could be improved by using a non linear approach for the dissipative forces.
\end{abstract}

Key words: Model identification; linear model; bridge; genetic algorithms; parallel selection; signal processing

\section{Introduction}

This paper is an updated and revised version of the conference paper [1]. The experimental model studied in this article was originally intended to reproduce approximately the transversal dynamic response of its prototype under earthquake excitation. Both the elastic response originated by minor earthquakes and the inelastic response due to strong motions was considered in the design of the model. The prototype is representative of a typical multi-span continuous-deck irregular bridge. The bridge had four identical straight spans of $50 \mathrm{~m}$ each. The three piers were respectively 14,7 and $21 \mathrm{~m}$ high, which corresponds to a rather irregular distribution.

The model was fabricated and dynamically tested on a shaking table at the facilities of the Earthquake Engineering Research Centre of the University of Bristol. The experiments were framed within the PREC8 (Prenormative Research for Eurocode 8) project, which was aimed to update the Eurocode 8 [2]. Several large-scale models of the same prototype were also developed and tested with equivalent earthquakes in different European laboratories, so as to allow the results to be compared. More details of this testing campaign can be found in reference[2-5]. Later on, the authors have developed a Finite Element (FE) model of the bridge. This FE model was updated on the basis of its natural frequencies, which were identified from modal tests carried out before the seismic ones [5].

\footnotetext{
${ }^{a}$ Corresponding author: david.bassir@utbm.fr
}

Studies on the effects of past strong earthquakes (Northridge 1994, and Kobe 1995) on steel structures have revealed some deficiencies in the modern seismic codes. The response of the structures in these earthquakes was good in terms of safety; only a few of them collapsed. The level of damage, however, was higher than that predicted by the codes in many cases $[6-8]$. As a consequence, the so-called performance-based approach has been recently proposed for the seismic design [9]. Several performance levels are considered in this new approach. At the Immediate Occupancy Level, only limited overall structural damage is allowed. The evaluation of this performance level is based on the structural seismic response, which is obtained through mathematical models, the structural displacements being the essential evaluation parameters. As only light structural damage is allowed at this level a linear elastic model with viscous damping is recommended for the structural analysis [9]. Recent studies [10], however, have proved that the calculated seismic response strongly depends on the modelling of the damping. This is due to the fact that only a small part of the energy is dissipated by plastic deformation at this level, most of it being dissipated by damping. The aforementioned reference only includes analytical simulation on single-degree-of-freedom models with different kinds of damping, and it is concluded that further research on this issue is needed.

The present article incorporates the experimental aspect to this research field taking advantage of the previous 


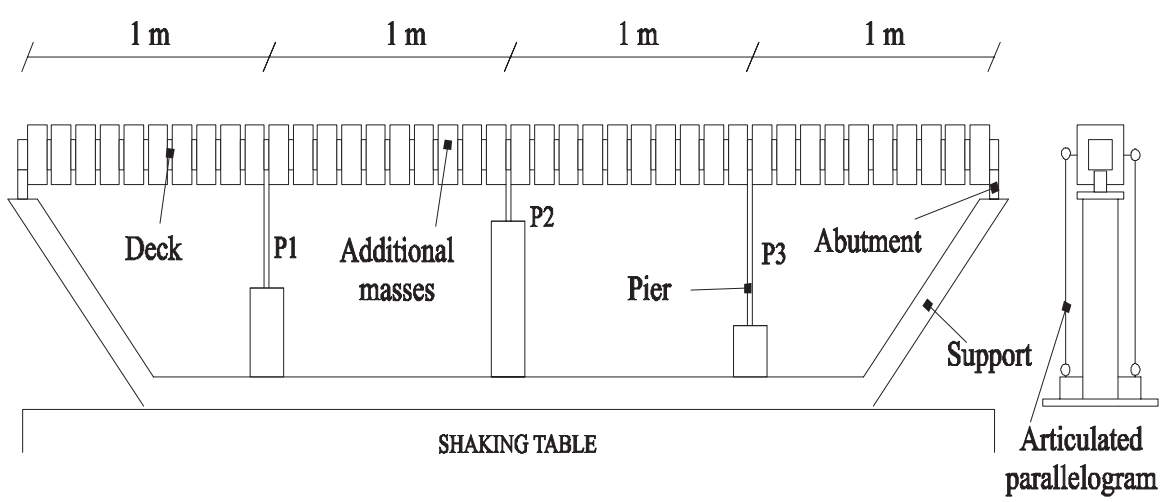

Fig. 1. Elevation of the experimental model.

tests on the small-scale bridge. The aim is to evaluate the accuracy of a linear viscous model to reproduce the preyielding seismic response of the actual bridge. For this purpose, an analytical model is proposed instead of the of the FE one, and fitted to the available seismic data in the time domain. Namely, the terms of the stiffness and damping matrices are directly obtained by minimizing the discrepancies between both the analytical model and the experimental model. As there is not a previous calibration of the damping coefficients in this case, and they can have a large interval of variation, a Genetic Algorithm (GA) has been selected for solving the optimization problem, so as to increase the probability of finding the global minimum solution. The seismic response of this calibrated analytical model is finally compared with the measured one. Besides, the terms of the stiffness matrix obtained in this process are likened to those of the previous FE model in order to evaluate the agreement between both approaches.

\section{Experimental model}

The model scaling was mainly conditioned by the characteristics of the earthquake simulator. In order not to exceed the maximum available length of the shaking table, a geometric scale factor of 50 was adopted. An artificial mass simulation model was chosen, with the acceleration scale factor set equal to one [5]. The adopted geometric scale meant that the materials used for the model had to be different from those of the prototype. Structural steel BS 4368 grade $43 \mathrm{c}$ was chosen instead of post-tensioned concrete for the model deck, and aluminium alloy grade 6082 T6, which was annealed before machining, was chosen instead of reinforced concrete for the model piers. Three models labelled as A, B, C, which correspond to different configurations of the piers, were used in the initial project. Configuration A was designed according to Eurocode 8, while configurations $\mathrm{B}$ and $\mathrm{C}$ were possible alternatives to the irregular issue. The former consist on reinforcing the shortest pier in order to reduce its ductility demand. In the latter configuration the taller piers were reinforced, so as to reduce the forces acting in the shortest pier. Only Configuration A has been considered for identification purposes herein.

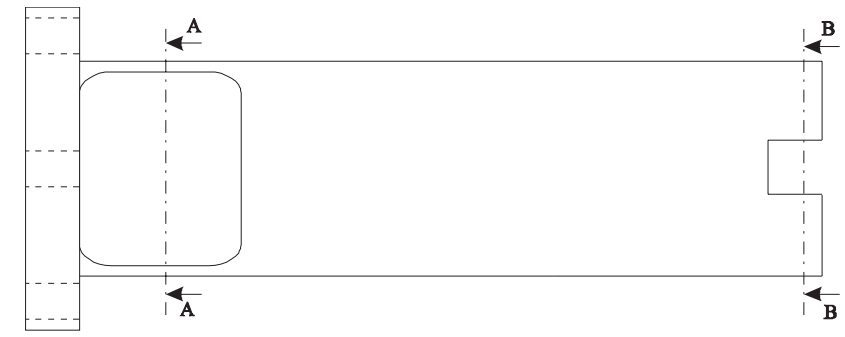

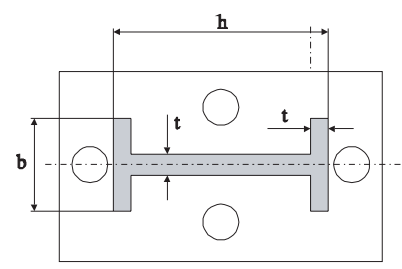

A-A

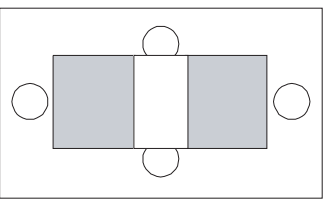

B-B
Fig. 2. Details of the piers.

\subsection{Deck}

The model deck was designed with a continuous square hollow section. A $60 \times 60 \mathrm{~mm}, 3.2 \mathrm{~mm}$ thick section was used, that had a similar second moment of area to that required for similarity between the prototype and model. Supplementary masses were added to the model, so as to attain mass similarity. These additional masses are distributed along the deck, and they consist of steel blocks bolted to it. In order to transmit the extra weight of the additional masses and to constrain the deck to translate only in the horizontal direction, articulated parallelograms are attached to the deck at the pier locations (Fig. 1).

\subsection{Piers}

The piers were designed with an I-beam section at the bottom, and a rectangular section for the remainder. Both sections had the same depth and width for each pier (Fig. 2). This design was intended to approximately reproduce the response of the prototype piers under vertical and transverse loads, acting simultaneously. 


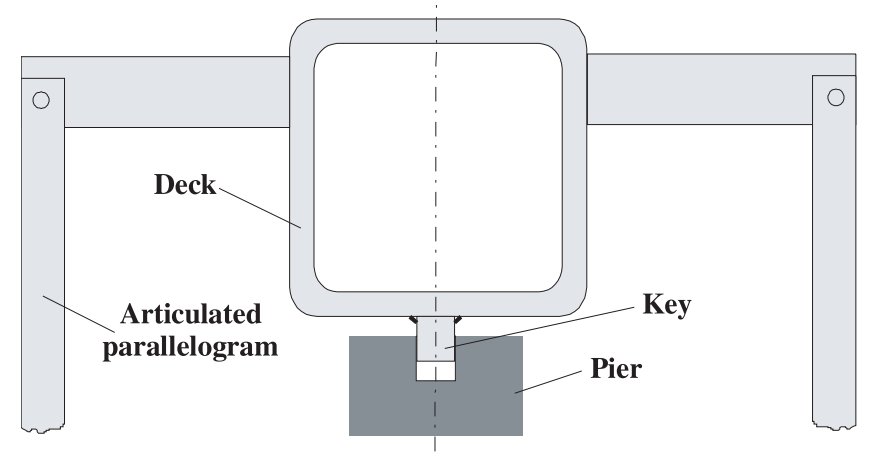

Fig. 3. Connection of the piers to the deck.

\subsection{Connections}

The deck ends hinged on the abutments through two vertical pins $20 \mathrm{~mm}$ in diameter. All of the piers had the same type of connection to the support including four $6 \mathrm{~mm}$ diameter bolts. The connections to the deck were also the same for all piers and consisted of square keys welded to the deck that fitted into slots in the piers. This ensured that the articulated parallelograms rather than the piers took the weight of the deck (Fig. 3).

\subsection{Testing}

In each configuration of the bridge, an initial modal test followed by several seismic tests was carried out. In the modal tests, the model was directly excited in the transverse direction by the shaking table with a low intensity random vibration. The response of the model was measured by an accelerometer that was placed at different positions on the deck and support using a magnetic base.

The model was also shaken in the transverse direction in the seismic tests. They consist of a series of earthquakes with the same time-history but increasing intensity, namely: $0.5,0.8,1.0,1.2$ and 2.0 times the design intensity. The reference earthquake was a synthetic one fitting the Eurocode 8 elastic response spectrum for medium soil conditions, had a maximum acceleration of $0.35 \mathrm{~g}$ and duration of 13 seconds at full scale.

During these tests, the absolute acceleration of the deck at the connection to the piers was measured by means of linear accelerometers. The absolute acceleration of the table was also recorded in all the configurations and tests. More details of the experimental model and testing can be found in reference [5].

\subsection{Signal pre-processing}

The analogue signals were converted into digital ones at sampling frequency of $1035 \mathrm{~Hz}$. An initial interval previous to loading was also recorded in order to check the quality of the signals. All the measurements of this initial part exhibit similar shape; an example is shown in

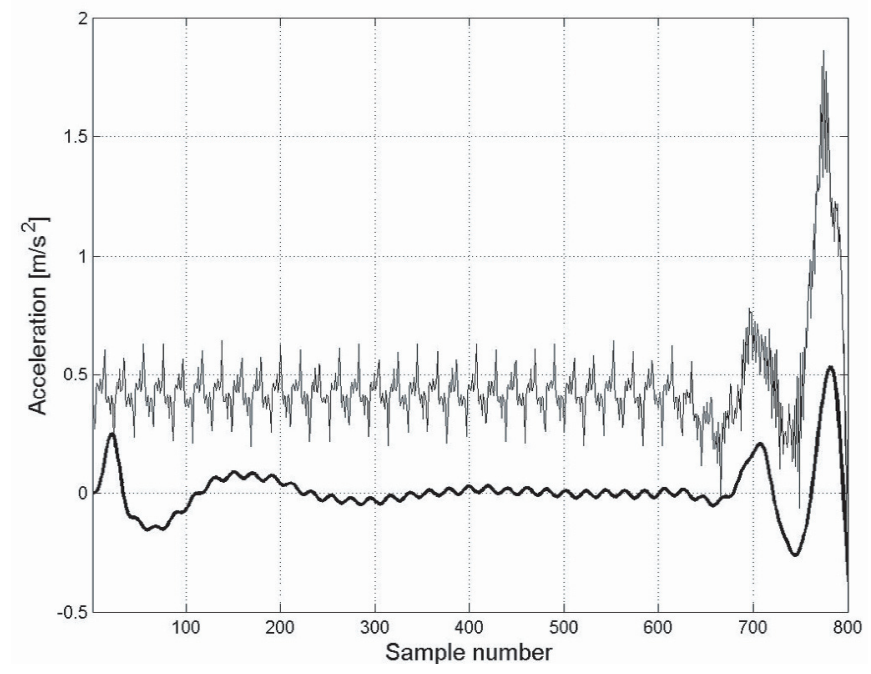

Fig. 4. Initial part of the raw signal (fine line) and the corresponding filtered one (thick line).

Figure 4. As it can be seen, the signal contains an offset and a sort of periodic high-frequency noise. As the offset is quite constant in this part, it was initially removed by subtracting the mean value of the signal in the complete sampling interval. The further integration of this acceleration signal, however, showed trends of the displacements with magnitude even higher than the pick-to-pick displacements. This means that the offset is not constant, but it changes during each test.

A band-pass filter, which is intended to remove both the low-frequency signal trend and the high-frequency noise, was eventually used. It was a four-order Butterworth filter with cut-off frequencies at 4.14 and $36.22 \mathrm{~Hz}$, which was applied to both the input and the output signals. These cut-off frequencies were selected as far as possible from the first natural frequencies of the model (11.6, 13.6 and $25.75 \mathrm{~Hz}$ ), which have the more significant influence in the seismic response of the bridge, seeking for a clean signal but retaining all the significant dynamic information of the raw one.

The filtered signal is shown in Figure 4 along with the raw one. It is visually evident that both the offset and the high-frequency noise are reduced. Nevertheless, the components of the noise within the filtering interval still remain in the pre-processed signal.

\section{Definition of the model and criterion of fitting}

\subsection{Analytical model}

The identification is based on the available experimental data induced by the lowest intensity earthquake ( $0.5 \times$ design intensity) carried out in the experiments. Under these conditions the bridge is expected to behave quasi elastically and with low displacements. Hence, an analytical linear model with viscous damping has been 
selected as a first approximation to reproduce the dynamic response of the experimental one. The mathematical formulation of the earthquake-induced response of the model is

$$
[M]\left\{\ddot{x}_{a}(t)\right\}+[C]\{\dot{x}(t)\}+[K]\{x(t)\}=0,
$$

where the vector $\{x\}$ represents the transversal displacements of the deck relative to the table at the connections to the piers. $\left\{\ddot{x}_{a}\right\}$ is the vector of absolute accelerations at the same Degrees of Freedom (dofs). $[M],[C],[K]$ are respectively the corresponding mass, damping and stiffness matrices.

It is assumed that the damping matrix is diagonal, and the stiffness matrix is symmetric according to the Betti's law. In order to avoid the identification process to be ill-conditioned, the mass matrix is set constant and equal to that obtained by dynamic condensation of the updated FE model of the bridge developed previously [5]. Summing up, the parameters of the model to be identified are the six independent terms of the stiffness matrix and the three terms of the leading diagonal of the damping matrix

\subsection{Objective and penalty functions}

The analytical model was calibrated by fitting its response to the experimental data in time domain. The available experimental data are the absolute acceleration of both the table $a_{g}$ and the deck $\left\{\ddot{x}_{a}\right\}$. From these, the acceleration of the deck relative to the table is obtained as follows

$$
\{\ddot{x}(t)\}=\left\{\ddot{x}_{a}(t)\right\}-\{1\} a_{g}(t) .
$$

Then, the velocity $\{\dot{x}\}$ and the displacement $\{x\}$ are in turn computed by numerical integration through the trapezium rule. The displacements predicted by the model $\left\{x^{\prime}\right\}$ are calculated from (1) on the basis of the measured absolute acceleration $\left\{\ddot{x}_{a}\right\}$ and the computed relative velocity $\{\dot{x}\}$

$$
\left\{x^{\prime}(t)\right\}=-[K]^{-1}\left([M]\left\{\ddot{x}_{a}(t)\right\}+[C]\{\dot{x}(t)\}\right) .
$$

A Normalized Mean Square Error (NMSE) is defined to quantify the discrepancies between the computed relative displacements $\{x\}$ and the predictions of the model $\left\{x^{\prime}\right\}$

$$
N M S E=\frac{1}{3} \sum_{i=1}^{3} \frac{\sum_{t=1}^{N}\left(x_{i}^{\prime}(t)-x_{i}(t)\right)^{2}}{\sum_{t=1}^{N}\left(x_{i}(t)\right)^{2}},
$$

which constitutes the objective function to be minimized.

The stiffness matrix is constrained to be positive definite during the minimization process, so as to obtain solutions with physical meaning. This is achieved by penalizing the non positive definite solutions. For this end, the eigenvalues of the stiffness matrix are computed at each stage of the minimization process. If the lowest eigenvalue is positive, which means that the stiffness matrix is positive definite, the solution is not penalized and the NMSE is calculated according equation (4). If the lowest eigenvalue is less than or equal to zero, then the solution is penalized by setting the NMSE equal to the summation of $\mathrm{NMSE}_{\max }$ (maximum admissible value obtained through the optimization process) and the absolute values of the eigenvalues (only the negative eigenvalues are considered in the summation).

A problem arising from the numerical integration of the relative acceleration (Eq. (2)) is the presence of linear and quadratic drifts in the calculated velocity and displacement, respectively. The magnitude of these drifts can be significant, and they might cause the identification process to fail. The drifts are due to unknown constant values of the acceleration and velocity that are not present into the signals. This problem is circumvented herein including these constant or initial values as parameters to be estimated in the minimization process.

\section{Minimization method}

As the objective of the identification consists on minimizing an error function that is highly multimodal, it's difficult to find one global solution. To overcome this problem, a robust identification procedure based on Genetic Algorithm with Parallel Selection (GAPS) coupled with a local search method has been developed [11]. Next subsections include a detailed description of such a procedure.

\subsection{Genetic algorithm with parallel selection}

Based on the theory of natural selection, the Genetic Algorithms (GAs) provide an alternative to traditional optimization techniques to locate the optimal solutions in a complex landscape. The theoretical foundations were first led by Holland in 1975 [12] and since that time, the number of applications and publications concerning GAs has increased with exponential manner. In 1989, Goldberg [13] gave to GAs their signs of nobility as an efficient and general method to overcome the complex optimization problems [14-20].

The principle of GAs is to simulate the evolution of one population of individuals to which different production operators (selection, crossover and mutation) are applied. As GAs start searching from different initial solutions, this gives them a global view of the problem. This global perspective prevents them to be trapped locally and allow them to explore all the search landscape. Such algorithms know the problem only through the value of the cost function and the values of the constraints. The behaviour of this algorithm is similar to a black box with several entries and one exit as described below in Figure 5.

The functioning of GAs can be carved in three main parts [21]: coding of parameters, genetic operators and choice of the objective function. 


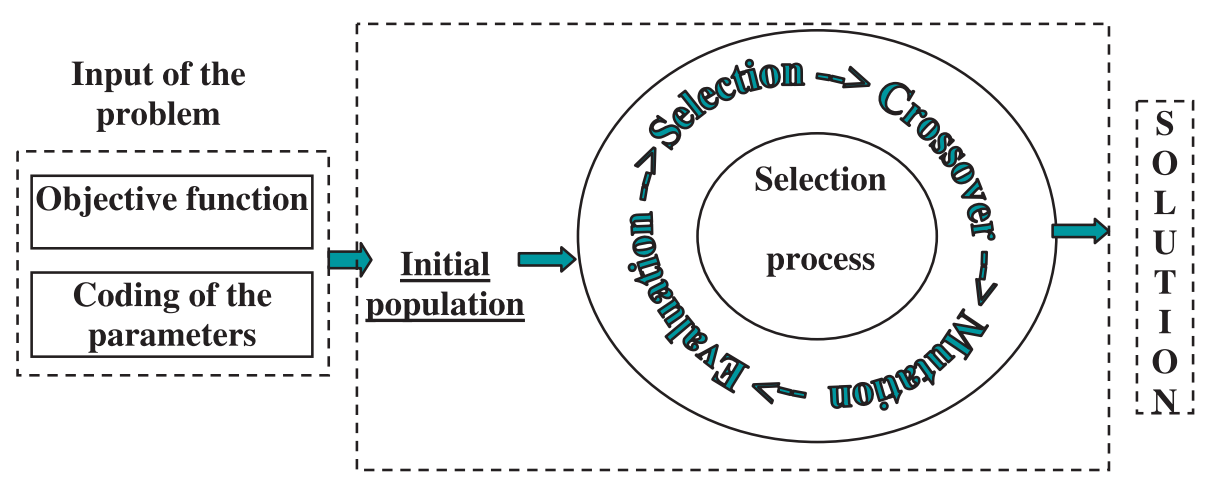

Fig. 5. Principle of a genetic algorithm.

The first problem to be faced during the use of the GAs is the representation of the individuals (coding of parameters). It is the manner each variable of the optimization problem is coded. The coding can be binary [17] coding or real coding $[17,19]$. The binary coding is the common representation that is most implemented in the GAs. For continuum variables, many authors [17, 20, 22] prefer using a real coding for their simplicity and efficiency for real problems. The real coding eliminate the difficulties of achieving arbitrary precision in decision variables and the Hamming cliff problem associated with binary string representation of real number $[13,17,19]$. In this article, the real coding [22] was chosen to be implemented in our program.

As concern the objective function, its definition is very important in the evolution process because GAs search in the landscapes by using only the discrete values of the objective function. If this function is not well defined, GAs cannot guaranty the location of the global solution.

Finally, the different operators used in GAs are the selection, the crossover and the mutation. These operators are widely described in the literature $[13,15,19,27]$.

In the following, we will introduce the basic ideas of the common operators with references to the literatures. Then, we will present the parallel selection introduced in our GAPS that is based on the advantage of the parallel processes in computing. The common selection method will be extended to the case of parallel calculation.

\subsection{Selection}

After creating randomly the first generation and evaluating the objective function for all individuals, the process of selection on the population is applied. There exist a number of selection operators in the literature of GAs. The main idea consists on choosing within a population $P$ a number $N$ of individuals (to create a mating pool) that are well adapted to survive and to follow their evolution to access to the next generation. The choice of keeping one individual is made by comparing its fitness function. One of the characteristics of the selection process is the selective pressure applied on the population. If this pressure is too strong, the diversity within the population is lost; whereas, if this pressure is too small, the behaviour of GAs becomes random.

In the following, focus on the tournament selection will be done. This selection method starts by choosing randomly $R$ individuals ( $R$ is the size of the tournament) of the population of $N$ individuals. The individual with a greatest fitness value wins the tournament and is saved for the next generation. This operation is repeated until $\mathrm{N} / 2$ individuals are obtained. It is entirely possible that some individuals participate in several tournaments: if they earn several times, they will have therefore straight to be copied several times. In this approach, the selection is strong enough, that is why one often reduce this pressure by decreasing the probability of selection in the tournament. This method has been integrated in GAPS as a pre-selection method.

\subsection{Crossover}

Crossover operator is applied next to the individuals of the mating pool. When the intermediate generation is half filled, it chooses randomly couples of individuals (parents) with a probability $P c$ of participation to the crossover. From a couple of parent, two children are created with the genetic patrimony of the parents, and then introduced in the population. In more concrete terms, the crossover operator favours the exploration of the research landscape. This exploration can be made by binary crossover (case of the binary coding) or by real crossover (case of the real coding).

In the case of real crossover, the same notion can be used either as for binary crossover or arithmetical approach. Thus the notion of point cut apparent more to a simple permutation of variables component without modifying the variable itself. In the case of arithmetical approach, the children $R_{1}^{\prime}$ and $R_{2}^{\prime}$ are generated by linear combination of the parents $R_{1}$ and $R_{2}$. This solution is created in the range of $\left[R_{1}-\alpha\left(R 2-R_{1}\right), \mathrm{R} 2+\alpha\left(R 2-R_{1}\right)\right]$ as follows:

$$
\left\{\begin{array}{l}
R_{1}^{\prime}=\left[\lambda R_{1}+(1-\lambda) R_{2}\right] \\
R_{2}^{\prime}=\left[\lambda R_{2}+(1-\lambda) R_{1}\right]
\end{array}\right.
$$

where $\lambda=\left((1+2 \alpha) P_{u}-\alpha\right)$ is the dispersion parameter, $\alpha$ define the range of the parents interval and $P_{u}$ a random value between 0 and 1 . 


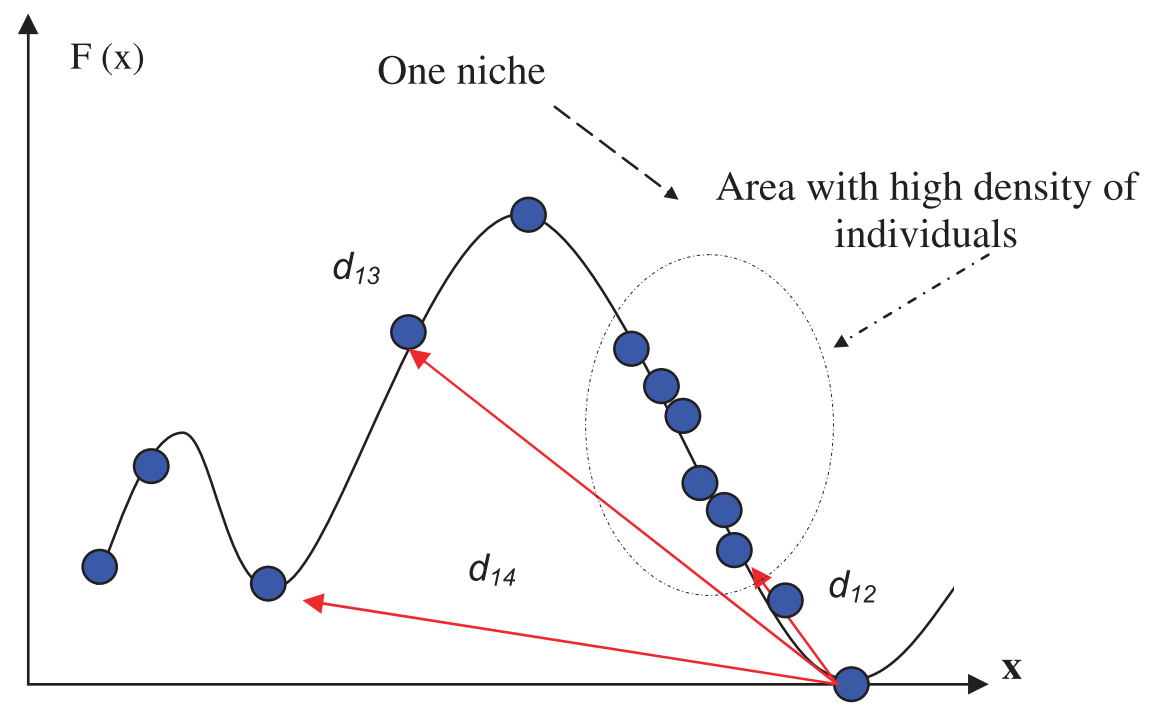

Fig. 6. Description of the relative distance with the sharing strategy.

A lot of studies about the dispersion parameter have been done specially by Deb [22-24] to understand and to control the dispersion of the individuals (children) in comparison with their parents. In the GAPS we have included the Deb approach in order to control the spread of the children. The children $R_{1}^{\prime}$ and $R_{2}^{\prime}$ are calculated as follows:

We choose one random value $P_{u}$ between 0 and 1 , then we evaluate the dispersion parameter $\beta$ (Eq. (6)) as a function of the parameter $n$ that define the probability of creating the new individuals close or far from the original individuals. If $n$ is small value (respectively if $n$ is a great value) we have a great probability to obtain the new individuals far from their parents (respectively close to the parents).

$$
\beta=\left\{\begin{array}{lr}
\left(2 P_{u}\right) \frac{1}{n+1} \quad \text { if } P_{u} \leqslant 0.5 \\
\left(\frac{0.5}{\left(1-P_{u}\right)}\right)^{\frac{1}{n+1}} \quad \text { otherwise }
\end{array}\right.
$$

Finally, the new solution $R_{1}^{\prime}$ and $R_{2}^{\prime}$ are calculated as follows:

$$
\begin{aligned}
& R_{1}^{\prime}=0.5\left[(1+\beta) R_{1}+(1-\beta) R_{2}\right], \\
& R_{2}^{\prime}=0.5\left[(1+\beta) R_{1}+(1-\beta) R_{2}\right] .
\end{aligned}
$$

We can find more details about the influence and the sensitivity of the individuals compared to their parent in the following articles [22] and [24].

\subsection{Mutation}

This operator intervenes in a population by modifying the genetic code of an individual. The need of mutation is to introduce diversity in the population, because the perpetual selection decreases gradually this diversity. Similarly to crossover operator, the mutation can be applied on a binary coding or on a real coding. In the case of a binary coding, the mutation operator inverts one bit of a chromosome from 1 to a 0 and vice versa with a small probability $P_{m}$. An individual can move depending on the importance of the bit inverted. In the case of a real coding, the mutation of an individual $R_{1}$ in a range of $\left[R_{1 \text { inf }}, R_{1 \text { sup }}\right]$ is done in general as follows:

$$
R_{1}^{\prime}=R_{1}+\delta\left(R_{1 \text { sup }}-R_{1 i n f}\right)
$$

where $\delta$ is the mutation parameter that defines the importance of the perturbation.

\subsection{Sharing method}

The concept of sharing is inspired by the nature. In a domain, two species can coexist and share the same resources. This idea [23] is introduced artificially in GAs through a sharing function $S$. This function consists in expelling some individuals from a zone of research that is reducing (example of the niches or zones of local minima) while the density of the population increases. The sharing function compares the relative distance $d_{i j}$ between two individuals $R_{i}$ and $R_{j}$ (see Fig. 6).

If $d_{i j}$ is greater or equal to $d$ ( $d$ namely niche radius), then $S$ is set equal to 0 . Whereas if $d_{i j}$ is less than $d$, then $S$ is set equal to a constant $c$ in the range $[0,1]$. Once this evaluation $S\left(R_{i}, R_{j}\right)$ is done for all individuals, a new fitness function $F$ is attributed to each one such as:

$$
F^{\prime}\left(R_{i}\right)=\frac{F\left(R_{i}\right)}{\sum_{j} S\left(R_{i}, R_{j}\right)} .
$$

Even if this idea increases the calculation time of GAs, it is very suitable in a lot concrete cases with many local optima because it makes stronger the exploration capabilities of GAs. That is why it has been introduced in the GAPS. 


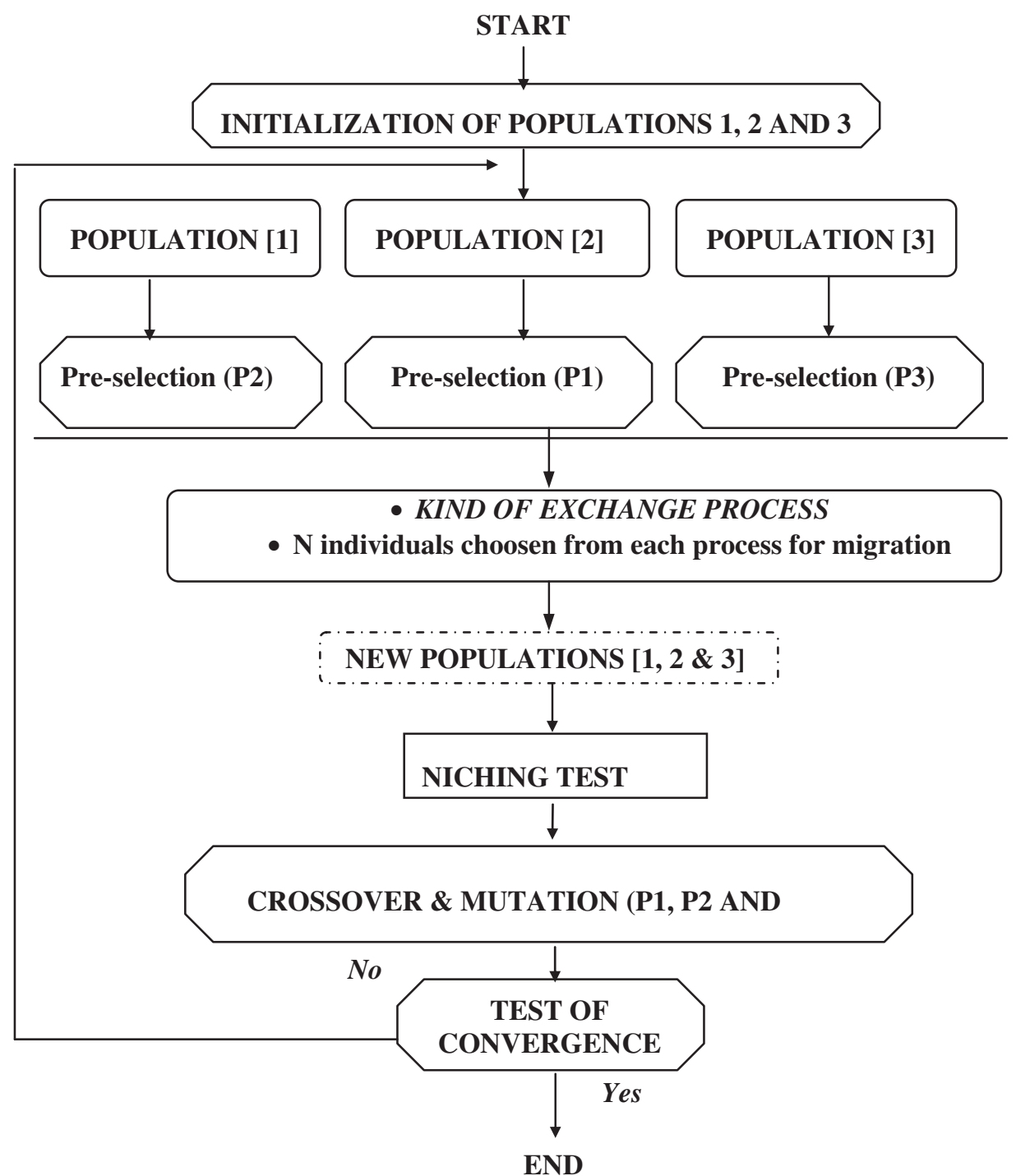

Fig. 7. Flow chart of the GAPS applied on 3 processes with an exchange of $2 \mathrm{~N}$ individuals.

\subsection{Parallel selection}

The Parallel Selection that is implemented in the GAPS is based on several populations that evaluate independently in different processes. The operator of selection occurs in a population by taking into account the other selections to introduce the diversity in each process. This diversity allows the pressure of selection to decrease and gives a chance to the other individuals to transmit their genetic patrimony. The first works related to parallelism of GAs consisted in simply running several GA programs in different process in parallel and waiting for the convergence of each process. Then, some researchers have leaned on the idea to establish parallelism in the operators of GAs themselves $[25,26]$. This parallelism consists on subdivide a population in several sub-populations that evolve not in the same global area, but in a reduced one. Selection, crossover and mutation operators are then applied to these sub-populations.
The parallel selection method (see Fig. 7) consists in introducing in a selection one or several individuals coming from other selection processes. This allows the different process to exchange information related to the area of research for a better exploration in all the landscape. This idea presents the following advantages. First, it decreases the number of evaluations of the objective function. Second, all the processes converge in the same area. In this operator of selection, the pressure of selection can be increased or decreased by increasing or by decreasing the number of individual to exchange between the different processes. Similarly, the choice of individuals that migrate to the other processes governs the rank of the diversity in the population.

\section{Application and analysis of results}

To apply the above identification strategy to the bridge, we have started by defining a large domain for each 

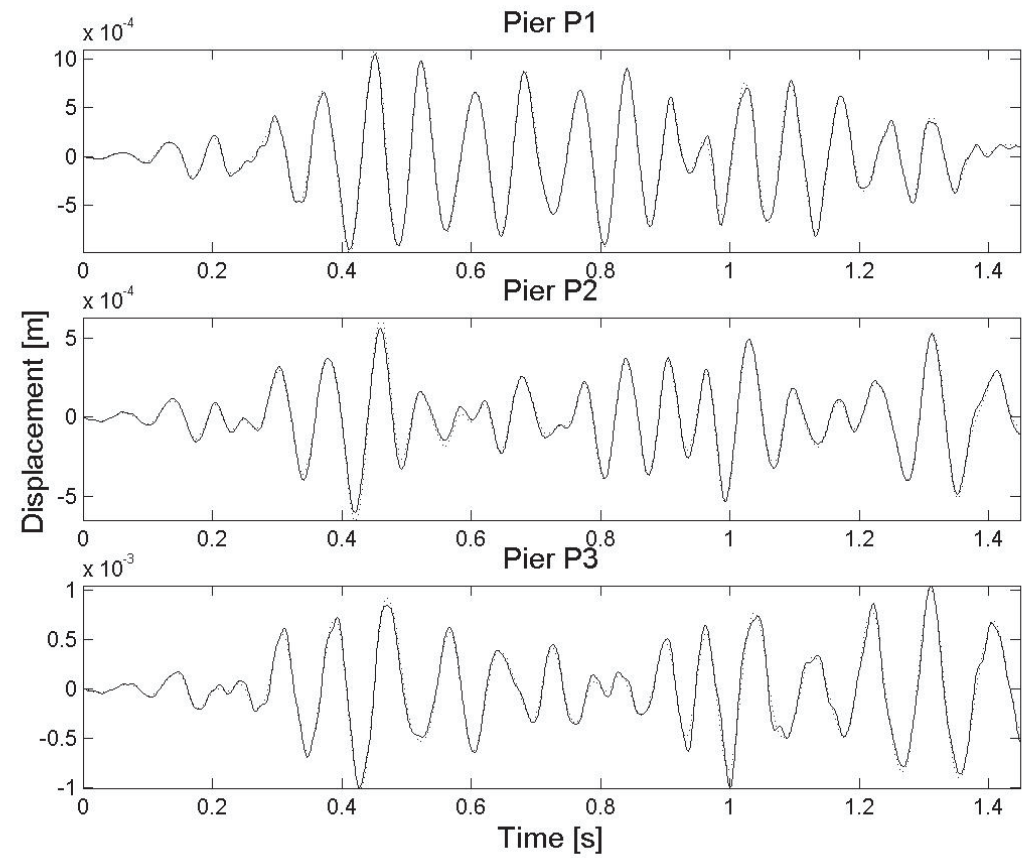

Fig. 8. Comparison between the displacements obtained by numerical integration of the measured accelerations (dotted line) and those predicted by the model (solid line).

parameter to be identified. For the coefficients $C_{\mathrm{ii}}$ of the damping matrix a range of variation between 0 and $10^{4}$ have been used. For the coefficients $K_{\mathrm{ij}}$ of the stiffness matrix a range of variation between $-10^{7}$ and $10^{7}$ have been used. Finally, as concern to the initial conditions of the velocity and the acceleration a range between -1 and 1 has been considered.

From the first GAPS tests, we have noted that the initial acceleration does not have much influence on the system response in this case. From this fact, then the number of parameters was reduced from fifteen to only twelve, the initial acceleration values being set equal to zero. The value of the objective function obtained with the GAPS at the convergence was $0.796 \%$ with a maximum number of iterations equals to 1200 generations and the time consuming equals to about 120 hours. The time of convergence can be explained by the initial intervals defined for the stiffness values $K_{\mathrm{ij}}$ that was too wide. The corresponding optimal solutions of the parameters are given below.

Stiffness matrix:

$$
[K]=\left[\begin{array}{lll}
1251132.51 & -987862.71 & 400913.73 \\
-987862.71 & 3164876.15 & -967343.67 \\
400913.73 & -967343.67 & 979652.18
\end{array}\right]
$$

Damping matrix:

$$
[C]=\left[\begin{array}{lll}
397.31 & 0 & 0 \\
0 & 1387.57 & 0 \\
0 & 0 & 121.56
\end{array}\right]
$$

Initial velocity:

$$
\left[\dot{x}_{0}\right]=\left[\begin{array}{l}
-1.03 e-3 \\
-8.34 e-3 \\
-1.22 e-3
\end{array}\right] .
$$

A comparison between the responses predicted by the model and those obtained directly by numerical integration of the measured accelerations are shown in Figure 8. In general, the predictions of the model are lower than those obtained by numerical integration for low displacements, and vice versa in the case of large displacements. Additionally, the absolute values of all the terms of the obtained stiffness matrix are lower than those obtained by FE model updating; the discrepancies being between $3 \%$ and $21 \%$ (see Fig. 9).

All these results indicate that the values of the "true" stiffness matrix diminish when the displacements increase, i.e., the behaviour of the bridge is not exactly linear. Thus, the response of the bridge could be more precisely described by a non linear model.

\section{Conclusions}

In this article, the properties of a small-scale irregular bridge and its dynamic tests have been described. An analytical linear model with viscous model is proposed to reproduce the response of the model to medium intensity seismic excitation. The parameters of the model are identified by minimizing the discrepancies between the response predicted by the model and that obtained in the 


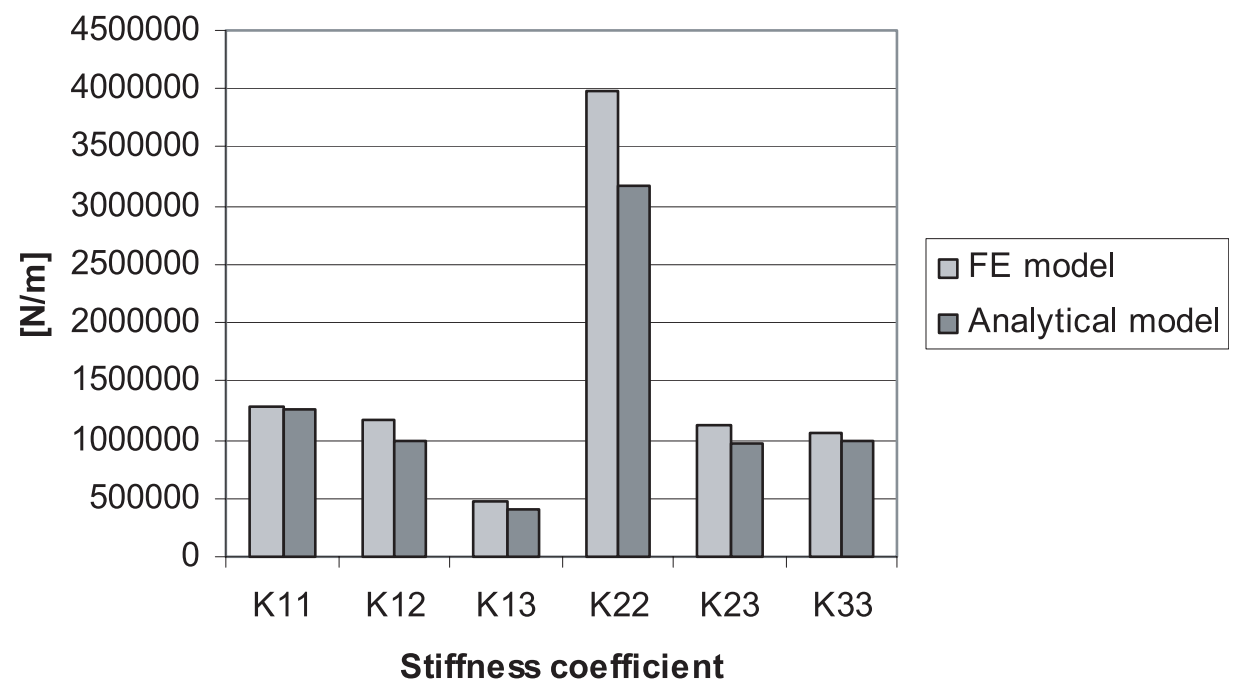

Fig. 9. Comparison between the stiffness coefficients corresponding to the updated FE model (Ref. [5]) and the analytical one

experiments in the time domain. A procedure based on GAPS has been developed for minimizing such discrepancies.

The application of the identification process shows that the strategies adopted for the signal pre-processing and GAPS are robust and efficient. The proposed model gives good dynamic predictions. The analysis of results, however, reveals that the model could be improved by using a non linear hysteretic scheme for the dissipative forces. This will be the matter of future studies on the bridge model.

Acknowledgements. The experimental part of this work was developed thanks to the ECOEST2 network project under the Human Capital and Mobility programme of the European Commission DGXII. The authors are grateful to it for their financial support. The tests took place in the facilities of the Earthquake Engineering Research Centre of the University of Bristol. The collaboration of the EERC staff, particularity Professor Severn, is greatly acknowledged. A part of this work was carried out under the AMERICO project (Multiscale Analysis Innovating Research for CFRP) directed by ONERA (French Aeronautics and Space Research Centre) and funded by the DGA/STTC (French Ministry of Defence), which is gratefully acknowledged.

\section{References}

1. J.L. Zapico, M.P. Gonzalez, D.H. Bassir, "Model identification of small-scale bridge using a genetic algorithm with parallel selection", in Proceedings of The Tenth International Conference on Civil, Structural and Environmental Engineering Computing, B.H.V. Topping, (Editor), Civil-Comp Press, Stirling, United Kingdom, paper 248, 2005

2. R.T. Severn, Proceedings of the Institution of Civil Engineers Structures \& Bridges, European experimental research in earthquake engineering for Eurocode 8 (1999) 134: $205-217$
3. V.A. Pinto, Pseudo-dynamic and shaking table tests on RC bridges. Report No 5, ECOEST \& PREC8, 1996

4. V.A. Pinto, G. Verzeletti, P. Pegon, G. Magonette, P. Negro, J. Guedes, Pseudo-dynamic testing of large-scale R/C bridges, Report EUR 16378 EN, ELSA, Ispra, Italy, 1996

5. J.L. Zapico, M.P. González, M.I. Friswell, C.A. Taylor, A.J. Crewe, Finite Element Model Updating of a Small Scale Bridge. Journal of Sound and Vibration 268, 9931012 (2003)

6. FEMA-355E., State of the art report on past performance of steel moment-frame buildings in earthquakes. Federal Emergency Management Agency, Washington, DC, 2000

7. M. Nakashima, K. Inoue, M. Tada, Classification of damage to steel buildings observer in the 1995 HyogokenNambu earthquake. Engineering Structures 20, 271-281 (1998)

8. S.A. Mahin, Lessons from damage to steel buildings during the Northridge earthquake. Engineering Structures 20, 261-270 (1998)

9. FEMA-350., Recommended seismic design criteria for new steel moment-frame buildings, Federal Emergency Management Agency, Washington, DC, 2000

10. D.V. Val, F. Segal, Effect of damping model on preyielding response of structures. Engineering Structures 27, 1968-1980 (2005)

11. D.H. Bassir, S. Carbillet, L.M. Boubakar, Algorithme génétique à sélection parallèle, Revue des Composites et Matériaux avancés, 15, 53-70 (2005)

12. J.H. Holland, Adaptation in natural and artificial systems, Ann Arbor: University of Michigan Press (1975)

13. D.E. Goldberg, Genetic algorithms in search, optimisation, and machine learning, New York: Addison-Wesley (1989)

14. L. Ljung, System Identification. New Jersey: Prentice Hall PTR (1999)

15. Z. Michalewicz, Genetic Algorithms + Data Structures $=$ Evolution, Springer-Verlag, Heidelberg (1994)

16. C.A.C. Coello, Use of the self-adaptive penalty approach for engineering optimization problems. Comput. Ind. 41, 113-127 (2000) 
17. C.Z. Janikow, Z. Michalewicz, An experimental comparison of binary and floating point representation in genetic algorithms, Proc. of the Fourth Int. Conference on genetic algorithms, R. Belew, L.B. Booker (Eds), (Morgan Kaufmman, San Mateo), 31-36, 1991.

18. K.A. DeJong, WM. Spears, An analysis of the interacting roles of population size and crossover in genetic algorithms, Proc., First workshop parallel problem solving from nature, Springer-Verlag, Berling, 38-47, 1990

19. A. Wright, Genetic algorithms for real parameter optimization. Foundations of genetic algorithms 1, G.J.E. Rawlin (Ed.) (Morgan Kaufmman, San Mateo), 205-218 (1991)

20. K. Deb, S. Gulati, Design of truss-structures for minimum weight using genetic algorithms. Finite Elem. Anal. Des. 37, 447-465 (2001)

21. K. Sastry, U.M. O'Reilly, D.E. Goldberg, Population sizing for genetic programming based upon decision making, IlliGAL, Report N. 2004028, April 2004

22. K. Deb, R.B. Agrawal, Simulated binary crossover for continuous search space. Complex Systems, 115-148 (1995)
23. K. Deb, D.E. Goldberg, An investigation of niche and species formation in genetic function optimization, in: J. D. Schaffer, ed., Proceedings of the Third International Conference on Genetic Algorithms (Morgan Kauffman, San Mateo), 42-50, 1989

24. K. Deb, H.G. Beyer, Self-Adaptive genetic algorithms with simulated binary crossover, Technical Report No. CI61/99, March 1999, Department of Computer Science/XI University of Dortmund, 44221 Dortmund, Germany

25. E.C. Paz, D.E. Goldberg, Efficient parallel genetic algorithms: theory and practice. Comput. Method. Appl. M. 186, 221-238 (2000)

26. N.J. Roudcliffe, Non linear genetic representations parallel problem solving from nature 2, R. Männer, B. Manderick (Ed.) Elsevier Science Publishers, Amsterdam, 259-268 (1992)

27. C.A.C. Coello, E.M. Montes, Constraint handling in genetic algorithms through the use of dominance-based tournament selection. Adv. Informatics 16, 193-203 (2002) 